\title{
Adaptive Pilot Allocation Algorithm for Pilot Contamination Mitigation in TDD Massive MIMO Systems
}

DOI:

10.1109/WCNC.2017.7925885

\section{Document Version}

Accepted author manuscript

Link to publication record in Manchester Research Explorer

\section{Citation for published version (APA):}

Alkhaled, M. H. M., Alsusa, E., \& Hamdi, K. (2017). Adaptive Pilot Allocation Algorithm for Pilot Contamination Mitigation in TDD Massive MIMO Systems. In 2017 IEEE Wireless Communications and Networking Conference IEEE. https://doi.org/10.1109/WCNC.2017.7925885

\section{Published in:}

2017 IEEE Wireless Communications and Networking Conference

\section{Citing this paper}

Please note that where the full-text provided on Manchester Research Explorer is the Author Accepted Manuscript or Proof version this may differ from the final Published version. If citing, it is advised that you check and use the publisher's definitive version.

\section{General rights}

Copyright and moral rights for the publications made accessible in the Research Explorer are retained by the authors and/or other copyright owners and it is a condition of accessing publications that users recognise and abide by the legal requirements associated with these rights.

\section{Takedown policy}

If you believe that this document breaches copyright please refer to the University of Manchester's Takedown Procedures [http://man.ac.uk/04Y6Bo] or contact uml.scholarlycommunications@manchester.ac.uk providing relevant details, so we can investigate your claim.

\section{OPEN ACCESS}




\title{
Adaptive Pilot Allocation Algorithm for Pilot Contamination Mitigation in TDD Massive MIMO Systems
}

\author{
Makram Alkhaled, Emad Alsusa, and Khairi A. Hamdi \\ School of Electrical and Electronic Engineering, \\ The University of Manchester, Manchester, M13 9PL, UK, \\ Email: makram.alkhaled@manchester.ac.uk,e.alsusa@manchester.ac.uk
}

\begin{abstract}
The pilot contamination problem is one of the major obstacles that limit the performance of time-division duplex (TDD) multicell massive multiple-input multiple-output (MIMO) systems. Pilot contamination results from the re-use of the same set of pilot sequences in the different cells of the system. In this paper, we propose an Adaptive Pilot Allocation (APA) algorithm for pilot contamination mitigation. The proposed algorithm divides the users in the system into two groups according to the inter-cell interference they cause to the other users in the system. To improve the system performance, the algorithm allocates orthogonal pilot sequences to the users that can cause high inter-cell interference. Simulation results show that the proposed algorithm outperforms the conventional (random) allocation method. Also it show that the proposed algorithm improves both the minimum and the mean achievable rates.
\end{abstract}

Index Terms-inter-cell interference, LSAS, massive MIMO, multicell MIMO, pilot contamination, pilot allocation.

\section{INTRODUCTION}

Massive MIMO systems have attracted a lot of attention from both academia and industry for their capabilities to provide high spectral and energy efficiency compared to conventional MIMO systems. In massive MIMO systems the BS is equipped with a very large number of service antennas (few hundreds) which are utilised to serve a smaller number of users (few tens) [1][2]. In TDD multicell massive MIMO systems, the users of each cell transmit pilot (training) sequences to their corresponding BSs. These sequences are pre-known to the BSs and orthogonal to each other. The BSs use the received pilot sequences to estimate the uplink channel matrix. Based on the estimated channel matrix, each BS builds its own detection matrix. The detection matrix is applied to the received signals vector to retrieve the uplink data symbols during the uplink phase. Moreover, based on the assumption of reciprocity between the uplink and the downlink channels, the estimated channel matrix is also utilized to build the precoding matrix which precodes the downlink data symbols during the downlink phase. Due to the scarcity of resources, the same set of pilot sequences used in one cell might be reused in the other cells. The re-use of the same set of pilot sequences results in a problem called pilot contamination. Pilot contamination simply means that the estimations of the channels between one BS and its own users are contaminated by the channels between that BS and the users of the other cells.

Pilot contamination limits the performance of TDD massive MIMO systems in both downlink and uplink. Thus, several methods have been proposed to mitigate or reduce the effect of pilot contamination. In [3], [4] and [5], a scheme based on a time-shifted (asynchronous) pilot transmission protocol is proposed to reduce the effect of pilot contamination. The proposed scheme partitions cells into several groups $A_{1}, A_{2}, \ldots, A_{\Gamma}$ and uses a time-shifted protocol in each group. The proposed scheme ensures that there is no pilot contamination among users from different groups. Thus, it achieves the same SINR as a system with frequency reuse factor of $\Gamma$.

A multicell minimum-mean squared error (MMSE) based precoding technique is proposed in [6]. In this technique, the precoding matrix of each $\mathrm{BS}$ is designed to minimize the sum of the squared error of its own users and interference to the users in all other cells. However, this precoding method should be combined with power allocation, power control, and scheduling when the sum rate is the performance metric of interest. In [7], a multicell massive MIMO system in which collaboration between cells is permitted is considered. Based on the assumptions made in [7], an outer multi-cellular precoding referred to as Pilot Contamination Precoding (PCP) is designed to eliminate the inter-cell interference resulting from the pilot contamination problem. The main idea of PCP is that each BS linearly combines messages for users in different cells that share the same pilot sequence. The combining coefficients of the proposed PCP depend on the slow fading coefficients between the users and the BSs only. Finally, by using conventional linear precoding that is based on the estimated fast fading coefficients, each BS independently transmits its PCPcombined symbols to its users. In [8], the multipath channel model for linear antenna arrays is considered. It is shown that users with mutually non-overlapping angle of arrival (AOA) probability density functions (PDFs) hardly contaminate each other even if they use the same pilot sequences. Based on this fact, the authors of [8] proposed a coordinated scheme for assigning identical pilot sequences only to users of this type. The proposed scheme can significantly reduce the intercell interference and increase the uplink and the downlink SINRs. 
In this paper we consider the problem of pilot contamination in multicell massive MIMO system and propose an algorithm that aims to improve the system performance under the destructive effect of pilot contamination. The basic idea of the proposed algorithm is to find the users that cause high inter-cell interference to the users in other cells. This is done by comparing their large scale fading coefficients with a predefined threshold value which can be adapted to achieve the optimum performance. After that, these users are assigned orthogonal pilot sequences that are not re-used by any other user in the system, which significantly reduces the inter-cell interference and improves the system performance compared to conventional (random) pilot allocation method.

The rest of the paper is organised as follows. The system model is illustrated in Section II. Section III describes the communication scheme of the system considered. The pilot contamination problem is discussed, and the proposed allocation algorithm is presented in Section IV. Numerical results are presented and discussed in Section V. Finally, conclusions are drawn in Section VI.

\section{System Model}

We consider the uplink of a multicell massive MIMO system with $L$ cells numbered $1,2, \ldots, L$ which are sharing the same frequency band. Each cell has one BS that is equipped with M service antennas to provide services to $K$ single antenna users. The users in each cell transmit data to their corresponding $\mathrm{BS}$ in the same time-frequency resources. Hence, the $M \times 1$ received signals vector at the $\mathrm{BS}$ of the $l$ th cell is given by

$$
\mathbf{y}_{l}=\sqrt{p_{u}} \sum_{i=1}^{L} \mathbf{G}_{l i} \mathbf{x}_{i}+\mathbf{n}_{l},
$$

where $p_{u}$ is the normalized transmit SNR, $\mathbf{x}_{i}$ is the $K \times 1$ vector of data symbols transmitted simultaneously by the $K$ users in the $i$ th cell, $\mathbf{n}_{l}$ is the $M \times 1$ vector of additive white zero-mean unit-variance Gaussian noise, and $\mathbf{G}_{l i}$ is the $M \times K$ channel matrix between the $K$ users in the $i$ th cell and the $M$ antennas of the $l$ th BS. The channel matrix $\mathbf{G}_{l i}$ models the independent fast fading, the geometrical attenuation, and the log-normal shadowing and can be represented as

$$
\mathbf{G}_{l i}=\mathbf{H}_{l i} \mathbf{D}_{l i}^{1 / 2}
$$

where $\mathbf{H}_{l i}$ is the $M \times K$ matrix of fast fading coefficients between the $K$ users of the $i$ th cell and the $M$ antennas of the $l$ th BS, i.e., $\left[\mathbf{H}_{l i}\right]_{m k}=h_{\text {limk }}$ is the fast fading coefficient between the $k$ th user in the $i$ th cell and the $m$ th antenna of the $l$ th $\mathrm{BS}$. The elements of $\mathbf{H}_{l i}$ matrix are assumed to be independent and identically distributed (i.i.d) zero-mean, circularly-symmetric complex Gaussian $\mathcal{C} \mathcal{N}(0,1)$ random variables and known to nobody. The matrix $\mathbf{D}_{l i}$ is a diagonal matrix of size $K \times K$, where $\left[\mathbf{D}_{l i}\right]_{k k}=\beta_{l i k}$ represents the large scale fading coefficient between the $k$ th user in the $i$ th cell and the $l$ th BS. We assume that the large scale fading coefficients are constant over many coherence intervals, independent over the antenna index $m$, and known to everybody.

\section{Communication Scheme}

The communication scheme for the system considered in this paper consists of two phases: training and channel estimation phase, and uplink data transmission phase. Next, we discuss these two phases in more details.

\section{A. Uplink Training and Channel Estimation}

We assume that an interval of length $\tau$ symbols is used for uplink training, and this interval is shorter than the channel coherence interval which is $T$ symbols in length. We further assume that all users in all cells simultaneously transmit pilot sequences of length $\tau \geq K$ symbols. Based on the received pilot sequences, the BSs estimate the uplink channel state information (CSI) and use these estimations to build the detection matrix. To minimize the intra-cell interference, the pilot sequences allocated to the users of one cell should be mutually orthogonal. Thus, the matrix $\boldsymbol{\Phi}_{i}$ of size $K \times \tau$ which represents the set of pilot sequences allocated to the $K$ users of the $i$ th cell should satisfies $\boldsymbol{\Phi}_{i} \boldsymbol{\Phi}_{i}^{\dagger}=\mathbf{I}_{K}$. The $M \times \tau$ matrix of received pilot signals at the $l$ th $\mathrm{BS}$ can be represented as

$$
\mathbf{Y}_{l}=\sqrt{p_{p}} \sum_{i=1}^{L} \mathbf{G}_{l i} \boldsymbol{\Phi}_{i}+\mathbf{N}_{l}
$$

where $p_{p}=\tau p_{u}$, and $\mathbf{N}_{l}$ is the $M \times \tau$ AWGN matrix at the $l$ th $\mathrm{BS}$ with i.i.d $\mathcal{C N}(0,1)$ elements. After receiving the pilot sequences, each BS estimates the channel vectors to its own users using one of the pilot based estimation method. In this paper, we assume that minimum mean square error (MMSE) estimation method is used, thus the estimated channel matrix $\hat{\mathbf{G}}_{l l}$ is given as [1]

$$
\hat{\mathbf{G}}_{l l}=\left(\sum_{i=1}^{L} \mathbf{G}_{l i}+\frac{1}{\sqrt{p_{p}}} \mathbf{W}_{l}\right) \tilde{\mathbf{D}}_{l l}
$$

where $\tilde{\mathbf{D}}_{l l}$ is a diagonal matrix with the $k$ th diagonal element $\left[\tilde{\mathbf{D}}_{l l}\right]_{k k}=\beta_{l l k}\left(\sum_{i=1}^{L} \beta_{l i k}+\frac{1}{p_{p}}\right)^{-1}$, and $\mathbf{W}_{l}=\mathbf{N}_{l} \boldsymbol{\Phi}_{l}^{\dagger}$.

\section{B. Uplink Data Transmission}

In this phase, the users in all cells transmit their data symbols to their corresponding BSs. Let $\mathbf{A}_{l}$ be the $M \times K$ linear detector matrix of the $l$ th BS which is built based on $\hat{\mathbf{G}}_{l l}$. In this paper, we assume that zero-forcing (ZF) linear detector is used, which is given as follows,

$$
\mathbf{A}_{l}=\hat{\mathbf{G}}_{l l}\left(\hat{\mathbf{G}}_{l l}^{\dagger} \hat{\mathbf{G}}_{l l}\right) \cdot{ }^{-1}
$$

The $l$ th BS multiplies the received signals vector given in (1) with the decoding matrix $\mathbf{A}_{l}^{\dagger}$ to retrieve the transmitted data symbols as follows

$$
\mathbf{z}_{l}=\mathbf{A}_{l}^{\dagger} \mathbf{y}_{l} .
$$

From (1) and (6), the received signals vector in the $l \mathrm{~h}$ BS after applying a linear detector can be represented as 


$$
\begin{aligned}
\mathbf{z}_{l} & =\sqrt{p_{u}} \mathbf{A}_{l}^{\dagger} \sum_{i=1}^{L} \mathbf{G}_{l i} \mathbf{x}_{i}+\mathbf{A}_{l}^{\dagger} \mathbf{n}_{l} \\
& =\sqrt{p_{u}} \mathbf{A}_{l}^{\dagger} \mathbf{G}_{l l} \mathbf{x}_{l}+\sqrt{p_{u}} \mathbf{A}_{l}^{\dagger} \sum_{\substack{i=1 \\
i \neq l}}^{L} \mathbf{G}_{l i} \mathbf{x}_{i}+\mathbf{A}_{l}^{\dagger} \mathbf{n}_{l} .
\end{aligned}
$$

Let $z_{l k}$ and $x_{i k}$ be the $k$ th elements of the $K \times 1$ vectors $\mathbf{z}_{l}$ and $\mathbf{x}_{i}$, respectively. Then, the signal of the $k$ th user at the $l$ the BS can be expressed as

$$
\begin{aligned}
z_{l k}= & \sqrt{p_{u}} \mathbf{a}_{l k}^{\dagger} \mathbf{g}_{l l k} x_{l k}+\sqrt{p_{u}} \sum_{\substack{j=1 \\
j \neq k}}^{K} \mathbf{a}_{l k}^{\dagger} \mathbf{g}_{l l j} x_{l j} \\
& +\sqrt{p_{u}} \sum_{\substack{i=1 \\
i \neq l}}^{L} \sum_{j=1}^{K} \mathbf{a}_{l k}^{\dagger} \mathbf{g}_{l i j} x_{i j}+\mathbf{a}_{l k}^{\dagger} \mathbf{n}_{l},
\end{aligned}
$$

where $\mathbf{a}_{l k}$ and $\mathbf{g}_{l l k}$ are the $k$ th columns of the matrices $\mathbf{A}_{l}$ and $\mathbf{G}_{l l}$, respectively. Hence, for the $k$ th user in the $l$ th cell, the uplink signal-to-interference-plus-noise ratio (SINR) can be calculated as

$$
\operatorname{SINR}_{l k}=\frac{p_{u}\left|\mathbf{a}_{l k}^{\dagger} \mathbf{g}_{l l k}\right|^{2}}{p_{u} \sum_{\substack{j=1 \\ j \neq k}}^{K}\left|\mathbf{a}_{l k}^{\dagger} \mathbf{g}_{l l j}\right|^{2}+p_{u} \sum_{\substack{i=1 \\ i \neq l}}^{L} \sum_{j=1}^{K}\left|\mathbf{a}_{l k}^{\dagger} \mathbf{g}_{l i j}\right|^{2}+\left\|\mathbf{a}_{l k}\right\|^{2}} .
$$

Assuming that the channel is ergodic and each codeword spans over a large number of realizations of the fast fading factor of the channel matrix $\mathbf{G}$ [1], the ergodic uplink achievable rate of the $k$ th user in the $l$ th cell can be expressed as

$$
R_{l k}=\mathbb{E}\left\{\log _{2}\left(1+\operatorname{SINR}_{l k}\right)\right\},
$$

and the spectral efficiency achieved by all the $K$ users in the $l$ th cell is given by

$$
S E_{l}=\sum_{k=1}^{K} R_{l k}
$$

\section{Pilot Contamination and Adaptive Pilot Allocation (APA) Algorithm}

\section{A. Pilot Contamination}

As massive MIMO systems are expected to be capable of serving large number of users simultaneously by sharing the same time-frequency resources among all system cells, allocating an orthogonal pilot sequence for every single user in the system becomes challenging. Thus, the same set of pilot sequences that is allocated to the users of one cell is re-used by the users in the other cells. Therefore, the channel estimate of the $k$ th user at the $l$ th BS becomes a linear combination of the channels to the $k$ th users in all the other cells, causing what is known in literature as pilot contamination. It has been extensively shown in the literature, for example [3],[9], and
[1], that with pilot contamination and when the number of BS antennas is very large $(M \rightarrow \infty)$, the uplink signal-tointerference-plus-noise ratio SINR of the $k$ th user in the $l$ th cell approaches the following limit

$$
S I N R_{l k} \rightarrow \frac{\beta_{l l k}}{\sum_{i \neq l} \beta_{l i k}}
$$

It is clear from (12) that the performance of the $k$ th user in the $l$ th cell is limited by the interference coming from the users in the other cell that are using the same pilot sequence. Based on this fact, we propose our adaptive pilot allocation (APA) algorithm.

\section{B. Adaptive Pilot Allocation (APA) Algorithm}

As the asymptotic SINR of a specific user is proportional to its large-scale fading coefficient and the large scale-fading coefficients of the users that are using the same pilot sequence but in different cells. This motivates us to propose allocating orthogonal pilot sequences to the users that are causing high interference to the users in the other cells. This can be done by dividing the users of each cell into two groups, namely, high interference users and low interference users. To start with the grouping process, we first assume that all the large-scale fading coefficients are made available to a centralised control unit which controls the pilot sequences allocation process, and we also assume that BS cooperation is invoked [7],[7], [4]. The APA algorithm starts by grouping the users of each cell into high or low interference users groups according to their large scale fading coefficients. For this purpose, we define the parameter $\gamma_{l k}$, which represents the sum of the large-scale fading coefficients from the $k$ th user in the $l$ th cell to the BSs of the other cells and given by

$$
\gamma_{l k}=\sum_{i=1, i \neq l}^{L} \beta_{i l k}, \quad \forall l=1 \ldots L, k=1 \ldots K
$$

Then the grouping process is implemented according to the following condition

$$
\gamma_{l k} \stackrel{?}{>} \rho \rightarrow\left\{\begin{array}{l}
\text { Yes } \rightarrow \text { High interference user } \\
\text { No } \rightarrow \text { Low interference user }
\end{array}\right.
$$

where $\rho$ is the user grouping threshold which is defined as

$$
\rho=\frac{\lambda}{K L} \sum_{l=1}^{L} \sum_{k=1}^{K} \gamma_{l k},
$$

where $\lambda$ is a system configuration parameter which can be adjusted to achieve the optimum performance. Finding the optimum value of $\lambda$ is discussed in more details in Sec. V.

For each cell and after the grouping process, the $K_{l}$ users of the $l$ th cell can be expressed as

$$
K_{l}=K_{l, l o w}+K_{l, h i g h},
$$

where $K_{l, l o w}=\operatorname{card}\left\{k: \gamma_{l k}<\rho\right\}$ is the number of low interference users, and $K_{l, h i g h}=\operatorname{card}\left\{k: \gamma_{l k} \geq \rho\right\}$ is the 
number of high interference users. After applying the grouping process to all the cells in the system, the total number of orthogonal pilot sequences required in the APA algorithm is given by

$$
K_{A P A}=K_{\text {low }}+K_{\text {high }},
$$

where $K_{\text {low }}=\max \left\{K_{l, l o w}, l=1,2, \ldots, L\right\}$ denotes the number of pilot sequences assigned to the users in the low interference groups in all cells, and $K_{\text {high }}=\sum_{l=1}^{L} K_{l, h i g h}$ is the number of pilot sequences assigned to the high interference users. Hence, the pilot sequences matrix $\boldsymbol{\Phi}_{A P A}$ which is of size $K_{A P A} \times K_{A P A}$ and satisfies $\boldsymbol{\Phi}_{A P A} \boldsymbol{\Phi}_{A P A}^{\dagger}=\mathbf{I}_{K_{A P A}}$ can be divided into

$$
\boldsymbol{\Phi}_{A P A}=\left[\boldsymbol{\Phi}_{l o w}^{T} \boldsymbol{\Phi}_{h i g h}^{T}\right]^{T},
$$

where $\boldsymbol{\Phi}_{\text {low }}$ contains the set of pilot sequences assigned to low interference users in all cells, and $\boldsymbol{\Phi}_{h i g h}$ contains the set of pilot sequences assigned to the high interference users, whereas $\boldsymbol{\Phi}_{\text {high }}$ can be further partitioned into $L$ partitions as

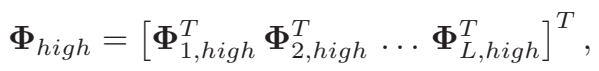

where $\boldsymbol{\Phi}_{l, h i g h}$ which is of size $K_{l, h i g h} \times K_{A P A}$ is the matrix of the orthogonal pilot sequences assigned to the users of the high interference group in the lth cell. Hence, the high interference users in all cells are assigned mutually orthogonal pilot sequences.

In Fig. 1, we show an example of the pilot allocation process with APA algorithm. We consider a system with 3 cells each associated with $K=6$ users. In order to entirely eliminate the pilot contamination effect, we need to use 18 orthogonal pilot sequences, which may degrade the system performance due to wasting the system resources during the channel estimation phase. On the other hand, and by applying the proposed APA algorithm, the number of required orthogonal pilot sequences is 11. Although the proposed APA algorithm consumes a bit more system resources compared to the conventional allocation method, the system performance can be significantly improved as will be shown in Sec. V.

\section{NuMERICAL RESULTS}

In this section, we evaluate the performance of the proposed APA algorithm by using Monte Carlo simulations. We consider a cellular massive MIMO system with $L=3$ cells, each has a central BS which is equipped with $M$ antennas and serving $K$ single antenna users that are randomly and uniformly distributed around it. We assume that the transmit SNR of each user is $p_{u}=10 \mathrm{~dB}$, and the channel coherence interval $T=200$ unless stated otherwise. The large-scale fading coefficients are generated according to [10]

$$
\beta_{l i k}=\frac{Z_{k}}{\left(d_{l i k} / R a d\right)^{v}},
$$

where $Z_{k}$ represents the shadow fading effect which is a lognormal random variable with standard deviation $\sigma=8 \mathrm{~dB}$, $d_{l i k}$ is the distance between the $k$ th user in the $i$ th cell and

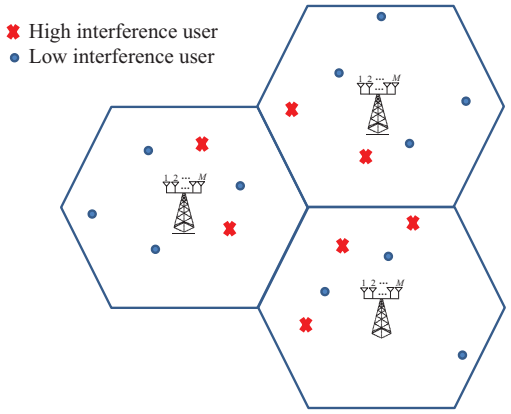

Figure 1. Example of the proposed APA algorithm.

the $l$ th BS, $R a d=500$ is the radius of each cell in meters, and $v=3$ is the path loss exponent.

Firstly, we investigate the effect of the system configuration parameter $\lambda$ on the system performance. Fig. 2 shows the uplink spectral efficiency versus the system configuration parameter $\lambda$ for different values of the channel coherence interval $T=100,200 \& 300$, when $K=14, M=128$. It is noticeable that $\lambda$ has significant impact on the system performance, thus it must be selected carefully to achieve optimum performance. When the channel coherence interval is small $(T=100)$, then $\lambda$ must be chosen large $(\lambda>2)$ to minimize the number of orthogonal pilot sequences required by APA algorithm, and maximize the system resources that are allocated for data transmission. On the other hand, when the channel coherence interval is large $(T=300)$, then $\lambda$ should be set to a small value $(\lambda=0.3)$ to ensure that more users are assigned orthogonal pilot sequences which in turn reduces the inter-cell interference and results in a better system performance. For moderate values of $T$, the optimum value of $\lambda$ is $\approx 1$. We should clarify here that the optimum value of $\lambda$ depends on other system parameters such as the number of users $K$, and the number of BS antennas $M$, and it can be found by utilizing one of the searching methods, for example, exhaustive search method, which is out of the scope of this paper.

Fig. V shows the UL spectral efficiency comparison of the proposed APA algorithm and the conventional (random) pilot allocation method [11],[12],[1] as a function of the number of BS antennas $M$, with $K=8$, and $\lambda=0.75$. Clearly, the proposed APA algorithm outperforms the conventional pilot allocation method. This due to the fact that the APA algorithm reduces the inter-cell interference by allocating orthogonal pilot sequences to the users that are causing high inter-cell interference to the users in the other cells.

Fig. 4 shows the cumulative distribution function (CDF) of the minimum achievable rate for $M=200, K=12$, and 


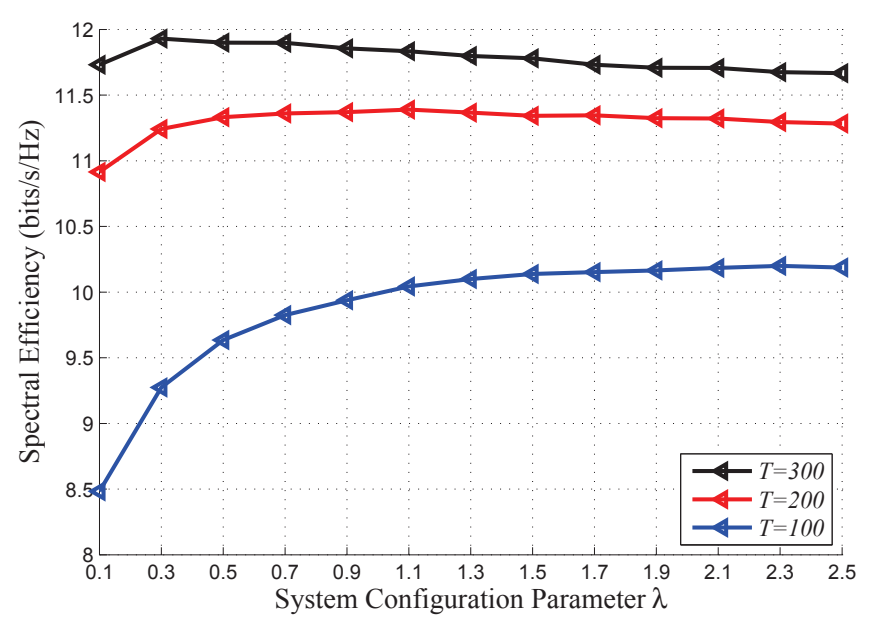

Figure 2. Uplink spectral efficiency versus system configuration parameter

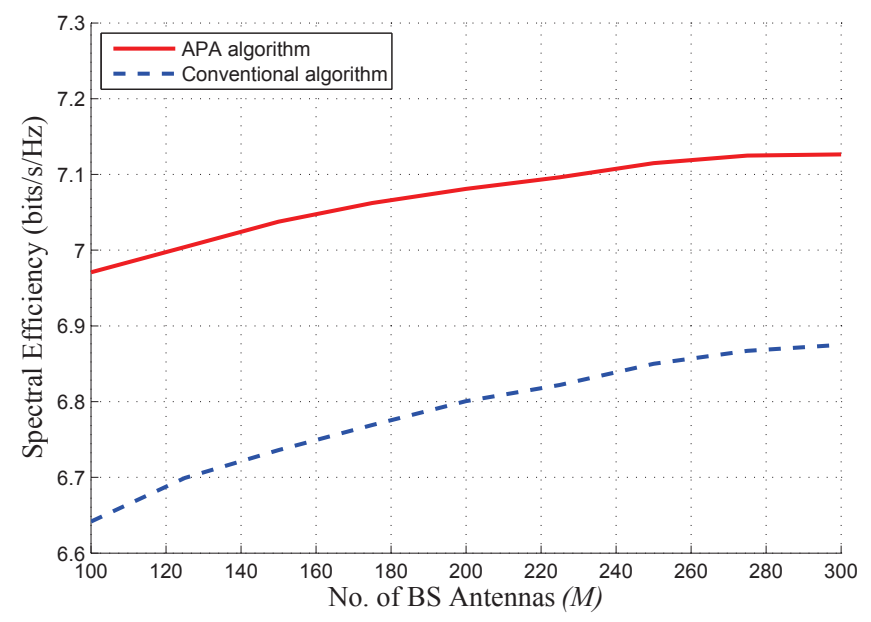

Figure 3. Uplink spectral efficiency for the proposed APA algorithm and conventional allocation method against $M$

$\lambda=1$. The APA algorithm has a significantly better minimum achievable rate performance than conventional allocation. This is clearly demonstrated by the fact that $50 \%$ of the time the minimum achievable rate of the conventional allocation is less than or equal to $0.15 \mathrm{bits} / \mathrm{s} / \mathrm{Hz}$ while for the APA algorithm it is approximately $0.55 \mathrm{bits} / \mathrm{s} / \mathrm{Hz}$. This improvement is caused by the reduced inter-cell interference when APA algorithm is used.

Fig. 5 shows the $\mathrm{CDF}$ of the mean achievable rate for $M=200, K=12$, and $\lambda=1$. The mean achievable rate attained by the APA algorithm is better than that of conventional allocation for $82 \%$ of the time. However, the conventional algorithm has a higher mean achievable rate for about $18 \%$ of the time. This is because in a bid to reduce inter-cell interference, the APA algorithm uses more system resources for the pilot allocation process which reduces system resources to some users with good channels hence such users perform better with the conventional allocation method.

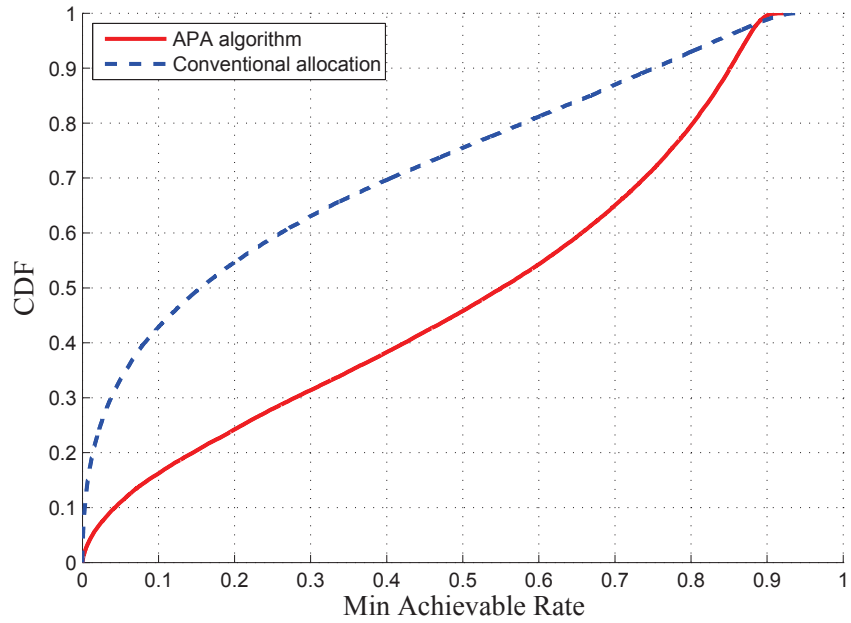

Figure 4. CDF of minimum achievable rate of the proposed APA algorithm and conventional allocation method.

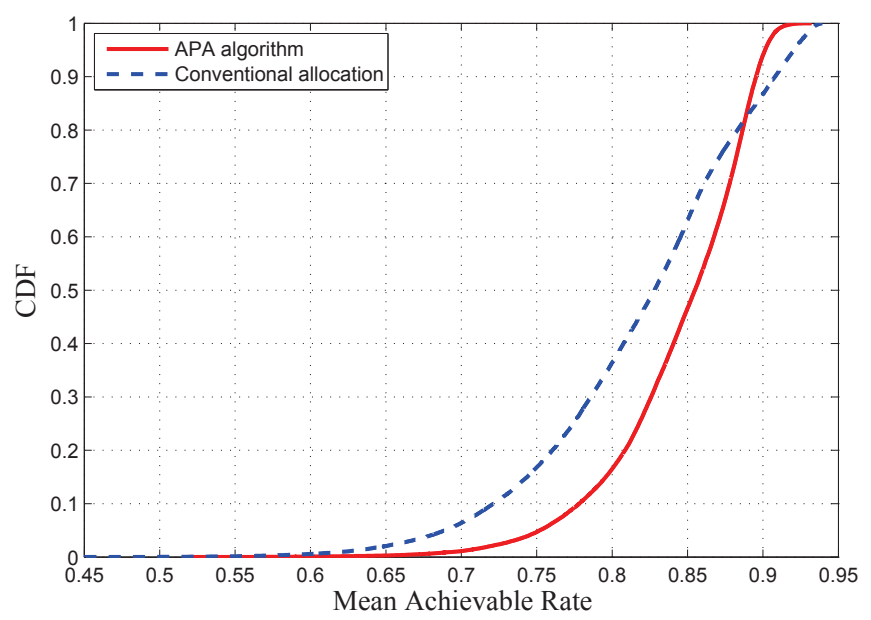

Figure 5. CDF of mean achievable rate of the proposed APA algorithm and conventional allocation method.

\section{CONCLUSIONS}

In this paper, we have considered the problem of pilot contamination in TDD multicell massive MIMO systems. In fact, pilot contamination which results from the re-use of the same set of pilot sequences in different cells limits the performance of multicell massive MIMO systems and prevents achieving high gains in both spectral and energy efficiencies. Thus, in this paper we have proposed the Adaptive Pilot Allocation (APA) algorithm, which aims to reduce the effect of pilot contamination and improve the system performance. Our numerical results have shown that although the proposed APA algorithm utilizes more system resources for the channel estimation process, it results in better system performance compared to conventional allocation method. Also, it significantly improves the minimum achievable rate by almost 3 folds compared to conventional allocation method for $50 \%$ of the time. 


\section{REFERENCES}

[1] H. Q. Ngo, E. Larsson, and T. Marzetta, "Energy and spectral efficiency of very large multiuser MIMO systems," IEEE Trans. Commun., vol. 61 , no. 4, Apr. 2013

[2] E. Larsson, O. Edfors, F. Tufvesson, and T. Marzetta, "Massive MIMO for next generation wireless systems," IEEE Commun. Mag., vol. 52 , no. 2, pp. 186-195, Feb. 2014.

[3] F. Fernandes, A. Ashikhmin, and T. Marzetta, "Inter-cell interference in noncooperative tdd large scale antenna systems," IEEE J. Sel. Areas Commun., vol. 31, no. 2, pp. 192-201, February 2013.

[4] K. Appaiah, A. Ashikhmin, and T. Marzetta, "Pilot contamination reduction in multi-user tdd systems," in Proc. IEEE Int. Conf. Commun. (ICC), May 2010, pp. 1-5.

[5] F. Fernandes, A. Ashikhmin, and T. Marzetta, "Interference reduction on cellular networks with large antenna arrays," in Proc. IEEE Int. Conf. Commun. (ICC), June 2012, pp. 1-5.

[6] J. Jose, A. Ashikhmin, T. Marzetta, and S. Vishwanath, "Pilot contamination and precoding in multi-cell tdd systems," IEEE Trans. Wireless Commun., vol. 10, no. 8, pp. 2640-2651, Aug. 2011.
[7] A. Ashikhmin and T. Marzetta, "Pilot contamination precoding in multicell large scale antenna systems," in IEEE International Symposium on Information Theory Proceedings (ISIT), July 2012, pp. 1137-1141.

[8] H. Yin, D. Gesbert, M. Filippou, and Y. Liu, "A coordinated approach to channel estimation in large-scale multiple-antenna systems," IEEE J. Sel. Areas Commun., vol. 31, pp. 264-273, February 2013.

[9] L. Lu, G. Li, A. Swindlehurst, A. Ashikhmin, and R. Zhang, "An overview of massive MIMO: Benefits and challenges," IEEE J. Sel. Topics Signal Process., vol. 8, no. 5, pp. 742-758, Oct. 2014.

[10] X. Zhu, L. Dai, and Z. Wang, "Graph coloring based pilot allocation to mitigate pilot contamination for multi-cell massive mimo systems," IEEE Communications Letters, vol. 19, no. 10, pp. 1842-1845, Oct 2015.

[11] T. Marzetta, "Noncooperative cellular wireless with unlimited numbers of base station antennas," IEEE Trans. Wireless Commun., vol. 9, no. 11, pp. 3590-3600, Nov. 2010

[12] H. Ngo, E. Larsson, and T. Marzetta, "The multicell multiuser mimo uplink with very large antenna arrays and a finite-dimensional channel," IEEE Trans. Commun., vol. 61, no. 6, pp. 2350-2361, Jun. 2013. 